\title{
IMPLEMENTACIÓN DE UN SISTEMA DE TRATAMIENTO CON PLASMA PARA GASES UTILIZANDO UNA CELDA DE DESCARGA DE BARRERA DIELÉCTRICA
}

\section{IMPLEMENTATION OF A SYSTEM OF TREATMENT WITH PLASMA FOR GASES USING A DIELECTRIC BARRIER DISCHARGE CELL}

\section{Resumen}

La utilización de las Descargas de Barrera de Dieléctrico, DBD, fue iniciada en Europa para la producción de ozono en el tratamiento de agua para el consumo humano. Desde entonces, el número de aplicaciones industriales de este tipo de descarga ha continuado aumentando. Actualmente las DBD son aplicadas con buenos resultados al control de la polución y tratamiento superficial de polímeros, con el fin de aumentar su facilidad de impresión y adhesión. La invención de la descarga luminiscente a presión atmosférica, basada en la DBD, ha impulsado trabajos teórico-experimentales para mejorar la comprensión de los mecanismos involucrados en ella. En este trabajo se realiza una descripción detallada del fenómeno DBD y se presenta la implementación de un sistema para el estudio del tratamiento con plasma de medios gaseosos. La funcionalidad del sistema desarrollado se verificó con aire, obteniéndose plasma visible a $12.31 \mathrm{kVy} 27.2 \mathrm{kHz}$. Los resultados encontrados en la experimentación son consistentes con los reportados en la literatura.

Palabras clave: descarga de barrera de dieléctrico, electrodos, microdescargas, plasma, reactor cilíndrico.
Eduin Yesid Mora Mendoza ${ }^{1}$ Armando Sarmiento Santos 2 Francy Mayoli Casallas Caicedo ${ }^{3}$

Recibido: junio 23 de 2014
ceptado: octubre 14 de 2014

The use of the Dielectric Barrier Discharge (DBD) was initiated in Europe for the production of ozone in the treatment of water for human consumption. From then, the number of industrial applications of this kind of discharge has continued increasing. Currently the DBDs are successfully applied to pollution control and polymers surface treatment in order to increase its printability and adhesion. The invention of the glow discharge at atmospheric pressure, which is based on the DBD, also theoretical studies have been conducted to achieve a better understanding of the mechanisms thereof. This paper presents a detailed description of the phenomenon DBD, and describes the development of a system for the study of the treatment with plasma of gaseous media. The functionality of the developed system was verified with air obtaining visible plasma at $12.31 \mathrm{kV}$ and $27.2 \mathrm{kHz}$. The results obtained in implementation are consistent with those reported in the literature.

Key words: dielectric barrier discharge, electrodes, micro-discharges, plasma, cylindrical reactor.

\footnotetext{
1 Ingeniero Electromecánico, Estudiante de Maestría en Metalurgia y Ciencias de los Materiales, Licenciatura en Tecnología, Universidad Pedagógica y Tecnológica de Colombia UPTC, E-mail: eduin.mora@uptc.edu.co

2 Ph.d. en Ciencias e Ingeniería de los Materiales, Escuela de Física, Universidad Pedagógica y Tecnológica de Colombia UPTC, E-mail: asarmiento.santos@uptc.edu.co

3 Ingeniero Electromecánico, Estudiante de Maestría en Metalurgia y Ciencias de los Materiales, Licenciatura en Tecnología Universidad Pedagógica y Tecnológica de Colombia UPTC, E-mail: francy.casallas@uptc.edu.co
} 


\section{Introducción}

Para las economías del mundo los procesos industriales son un importante componente, son generadores de progreso. Sin embargo, en la fabricación de algunos productos se generan poluciones en forma de emisiones de Dióxido de Carbono ( $\left.\mathrm{CO}_{2}\right)$, Dióxido de Azufre $\left(\mathrm{SO}_{2}\right)$, Dióxido de Nitrógeno $\left(\mathrm{NO}_{2}\right)$ entre otros, contribuyendo estos en buena medida al calentamiento global.

De lo anterior nace la necesidad de plantear soluciones que permitan tratar estos gases sin torpedear el desarrollo socioeconómico. Una de las posibilidades es la utilización del arreglo de descarga de barrera de dieléctrico, el cual realiza descargas eléctricas a grandes tensiones creando un campo eléctrico que es capaz de romper las moléculas del gas bajo tratamiento, con el fin de convertirlo en otras sustancias las cuales podrían ser menos agresivas con el medio ambiente. En este trabajo se reporta la implementación de una celda de Descarga de Barrera Dieléctrica, DBD, la cual es base para un sistema a nivel de laboratorio, que permite realizar experimentos en aplicaciones de tratamiento para gases de efecto invernadero.

En la primera parte del documento se presenta una descripción general de la DBD, la forma de generarse, sus características más importantes y algunas de sus aplicaciones. En la segunda parte se presenta la implementación del sistema DBD, el cual consta de un reactor cilíndrico acoplado a la línea de gases así como una fuente de tensión eléctrica de alto voltaje y alta frecuencia; para la selección y/o fabricación de los elementos se tomó como base lo reportado en la literatura. Una vez implementado, el sistema se puso a prueba con aire, generando descargas que permitieron establecer los parámetros adecuados para obtener una descarga DBD estable.

\section{Características y aplicaciones de las DBD}

\subsection{Requerimientos de la técnica}

La primera descarga de barrera de dieléctrico,
Dielectric Barrier Discharge: DBD, también llamada descarga silenciosa o descarga de barrera, fue realizada por Siemens en 1857 (Hippler et al., 2008 p.439). El dieléctrico cumple dos funciones: prevenir la formación de chispas y limitar la corriente eléctrica, empleando fuentes de potencia con pulsos de corta duración.

Las DBD usualmente operan a frecuencias de entre 50 y $500 \mathrm{kHz}$, teniendo numerosas aplicaciones debido a que operan en condiciones de no equilibrio a presión atmosférica para diferentes gases, incluido el aire. Una ventaja importante es que la potencia demandada normalmente no supera unos cuantos kilovatios, sin tener necesidad de usar fuentes de energía pulsadas sofisticadas (Fridman, 2008). Otra ventaja importante con relación a las descargas en corriente directa es que al utilizar altas frecuencias se promueve la movilidad de electrones con niveles de tensión más bajos (Figueroa, 2010).

El gap o región de descarga en la DBD se encuentra entre una o más capas dieléctricas, las cuales están colocadas entre los electrodos metálicos. En la figura 1 se ilustran dos configuraciones específicas de DBD, plana y cilíndrica.

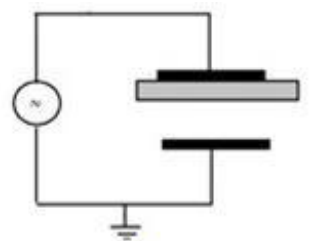

(a)

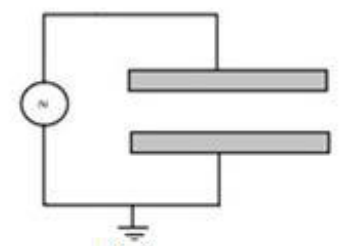

(c)
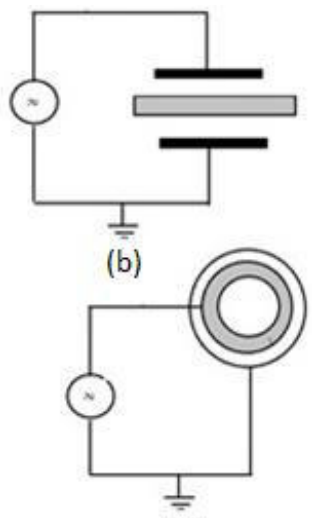

(d)
Figura 1. Diferentes configuraciones de la DBD: Plana (a), (b), (c), cilíndrica (d) (Fridman, 2008 p. 238)

Las distancias típicas en los espacios de descarga varía desde $0,1 \mathrm{~mm}$ hasta varios $\mathrm{cm}$. Si el espacio de la DBD es de unos pocos $\mathrm{mm}$, la tensión de manejo en $A C$ requerida con frecuencias de entre $500 \mathrm{~Hz}$ a $500 \mathrm{kHz}$ es de aproximadamente $10 \mathrm{kV}$ a 
presión atmosférica. Es precisamente debido a que existe material dieléctrico entre ambos electrodos, que este tipo de descargas requieren voltajes cambiantes para su operación, ya que al tratarse de material aislante no se permite el paso de la corriente directa, en cambio, en corriente alterna esta barrera solo es una limitante pero no un impedimento (Figueroa, 2010 p. 32). Por lo anterior, las DBD operan desde frecuencias de línea hasta frecuencias del orden de los mega Hertz; para frecuencias mayores, la limitación de corriente de la barrera dieléctrica no está asegurada (Figueroa, 2010).

El reactor para este tipo de sistemas normalmente está compuesto por la barrera dieléctrica, las tapas laterales y los electrodos interno y externo. La barrera dieléctrica puede ser de vidrio, cuarzo o cerámica (Fridman, 2008). La geometría más utilizada en la DBD es de tipo cilíndrico con el fin de evitar efectos de borde, los cuales reducen el efecto ionizante. En esta configuración se emplean principalmente electrodos de cobre o acero inoxidable en forma de varilla maciza para el interior que, a su vez, sirve de electrodo de alta tensión; también se pueden usar electrodos en forma de malla, la cual permite ver el plasma conseguido; otra alternativa son los electrodos de tubo hueco para el electrodo exterior, el cual a su vez es tomado como electrodo a tierra.

Para la medición y el análisis de las magnitudes eléctricas se utilizan instrumentos como osciloscopios y multímetros, que permiten cuantificar y cualificar la tensión, la corriente, la frecuencia y la potencia eléctrica de la descarga. Así mismo, los flujos de los gases sometidos a estudio se miden con flujómetros específicos para cada gas; éstos flujómetros, junto con los reguladores respectivos, permiten manejar las cantidades adecuadas para la experimentación, la cual tiene como principal objetivo el encontrar y mantener un plasma estable.

\subsection{Aplicaciones de los sistemas DBD}

Dentro de las principales aplicaciones desarrolladas para el uso o tratamiento de gases utilizando el arreglo DBD están:

\subsubsection{Generación de ozono}

La generación industrial de ozono utilizando DBD tiene una larga tradición. Hoy día, muchas plantas para tratamiento de agua a lo largo del mundo usan generadores de ozono utilizando la DBD para convertir químicamente moléculas diatómicas de oxígeno $\mathrm{O}_{2}$, en moléculas triatómicas más activas de ozono Оз (Hippler et al., 2008 p. 450). En muchos de los generadores pequeños de ozono, diferentes parámetros de operación son optimizados cuando el ozono es generado desde el aire; en este caso el nitrógeno no puede ser considerado como un gas de arrastre pasivo. Por el contrario la excitación y disociación de moléculas de nitrógeno promueven la formación de átomos de oxígeno adicionales que pueden ayudar a la formación de ozono ( Eliasson y Kogelschatz,1988; Weschler et al.,1992; Sillman et al.,1990).

\subsubsection{Láseres de $\mathrm{CO}_{2}$ de alta potencia}

Después de los experimentos iniciales de laboratorio con láseres de $\mathrm{CO}_{2}$ pulsados, realizados por Ishchenko (1978) y Christensen (1979), un láser de alta potencia industrial fue desarrollado por Yagi y Tabata (1996) en la corporación eléctrica Mitsubishi, a partir de electrodos de metal plano refrigerados por agua, cubiertos por vidrio o alúmina con una separación entre ellos de 20 a $50 \mathrm{~mm}$. A altas velocidades el flujo de gas pasa el espacio de descarga a razón de entre 20 y $50 \mathrm{~m} / \mathrm{s}$ para remover el calor y estabilizar la descarga. Se utilizan mezclas de gas de $\mathrm{CO}_{2}, \mathrm{~N}_{2}$ y He a presión de 5 a $20 \mathrm{Kpa}$, siendo la frecuencia de operación de aproximadamente $170 \mathrm{kHz}$ (Hippler et al., 2008 p.452).

\subsubsection{Control de polución}

Un gran número de investigaciones han sido desarrolladas no solamente para tratar óxidos de nitrógeno y óxidos de azufre en gases fluidos, sino también en la descomposición de compuestos 
orgánicos volátiles, como hidrocarburos, clorocarburos y clorofluorocarburos. La contaminación del aire con hidrocarburos o vapores solventes orgánicos ocurre en muchos procesos industriales, en procesos químicos para procesamiento de semiconductores, así como en el tratamiento mismo del agua. Algunos trabajos relacionados han sido publicados por Penetrantey Shultheis (1993) por Ruan et al., (1999) y por Sosa et al., (2009).

\subsection{Características físicas de las descargas}

En la mayoría de los casos las DBD no son uniformes y consisten en numerosas microdescargas construidas desde los canales ionizados delgados, streamers en inglés, y distribuidos en el espacio de la descarga, como se muestra en la figura 2 (Fridman, 2008 p. 164).

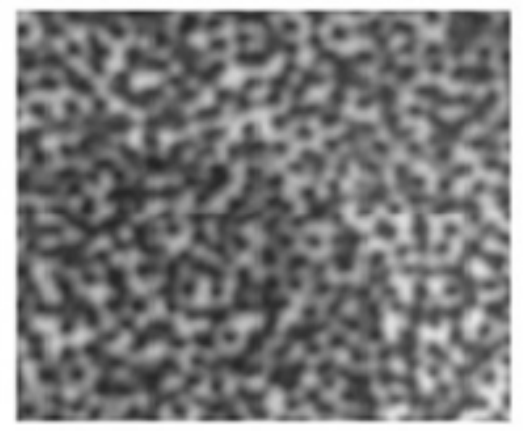

Figura 2. Imagen típica de numerosas microdescargas, distribuidas dentro del espacio de descarga (Fridman, 2008 p. 164)

La ruptura y desarrollo de la microdescarga ha sido estudiada por varios autores (Givalov y Pietsch, 1992; Reitz y Julich, 1992), mostrando los cuatro estados típicos que se presentan en la figura 3. Si el campo eléctrico en el espacio del gas es lo suficientemente grande para iniciar la avalancha, la ruptura comienza con la fase de Townsend, formándose un canal conductor o filamento. Nuevas cargas son transferidas a través del canal y acumuladas en la superficie dieléctrica. La tensión a través del filamento es compensada y la descarga se extingue (fase 4). La descarga completa toma alrededor de $1 \mathrm{~ns}$ (Hippler et al., 2008 p. 440)

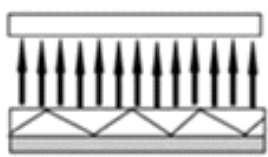

1 Fase Townsend

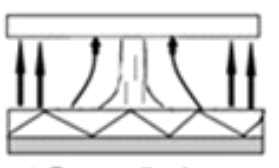

3 Desarrollo de una Capa Catódica

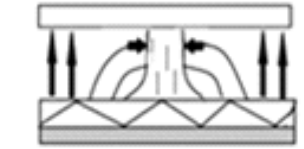

2 Ionización o Fase Streamer

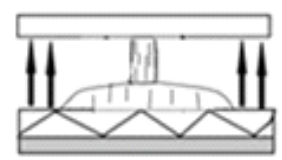

4 Extinción y fase de Recubrimiento
Figura 3. Desarrollo de la microdescarga (Hippler et al., 2008 p. 440)

En la figura 4 se aprecia la característica de Lichtenberg tomada de una fotografía de un montaje experimental (Salge, 1996). La imagen fue creada desde el frente del espacio de descarga y una placa de vidrio actuó como barrera de descarga.

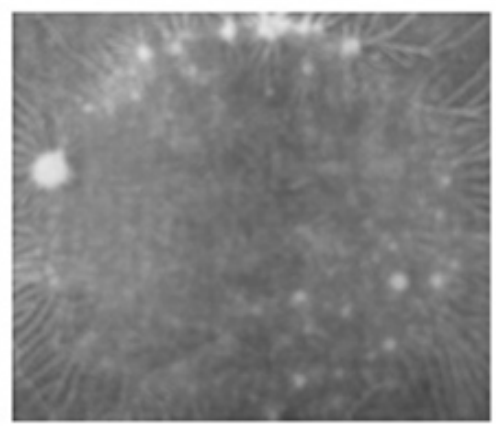

Figura 4. Fotografía que muestra la característica de Lichtenberg (Salge, 1996)

La formación de cada microdescarga depende de diferentes condiciones. Incluso aplicando señales de tensión con pulsos iguales, no necesariamente hacen que las microdescargas inicien al mismo tiempo. En consecuencia también las propiedades de la microdescarga: tiempo de conducción de corriente, dimensiones del canal, calentamiento del canal e intensidad de radiación, son diferentes. El desarrollo de nuevas descargas está determinado principalmente por las cargas depositadas sobre la superficie del dieléctrico, junto con la tensión aplicada (Hippler et al., 2008 p. 441).

Las microdescargas se caracterizan a presión 
atmosférica, teniendo estas una duración de unos pocos ns, alcanzando densidades de corriente de $100 \mathrm{~A} / \mathrm{cm}^{2}$ en filamentos cilíndricos delgados de cerca de 100 micrómetros de radio. En esos canales de descarga y en descargas de superficie, pertenecientes a condiciones de plasma de no equilibrio, la caracterización puede ser obtenida como transcientes de descargas luminiscentes de alta presión. Esas condiciones son ideales para la generación de iones y de especies moleculares y atómicas excitadas que pueden ser utilizadas para reacciones de superficie y volumen y para eficiente generación de radiación de longitud de onda selectiva (Hippler et al., 2008 p. 441).

\section{Implementación del sistema de DBD}

\subsection{Diseño y fabricación del reactor}

En el desarrollo del reactor se incluyeron las siguientes partes:

\subsubsection{Electrodo externo}

Para este electrodo que recubre el dieléctrico se utilizó una lámina de acero inoxidable, debido a que este presenta una buena conductividad eléctrica y buenas propiedades mecánicas para afrontar el proceso de conformación cilíndrica; las dimensiones de la lámina son $11 \times 10 \mathrm{~cm}$, la cual fue curvada y soldada, según se aprecia en la figura 5. En varios trabajos se ha reportado el uso del acero inoxidable como material para los electrodos (Kyung et al., 2012; Indarto, 2007; Sarmiento et al., 2007; Chong-Lin et al., 2008).

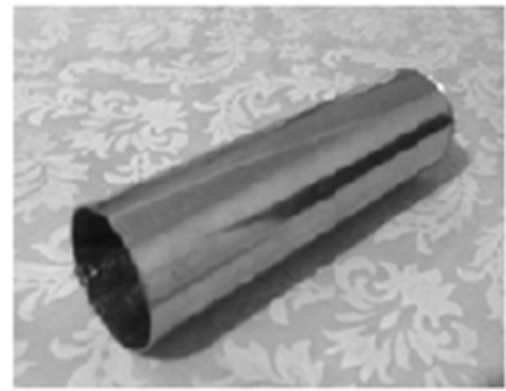

Figura 5. Pieza de acero inoxidable como electrodo externo

\subsubsection{Electrodo interno}

Como electrodo interno se utilizó una varilla de 1/4 pulgadas de acero al carbono A36, la cual fue sometida a remoción de óxidos y a un proceso de cromado electroquímico durante 20 minutos; este último proceso permite cubrir toda la superficie de la varilla con este metal, lo cual facilita la conducción eléctrica; Antonius Indarto (2007), utiliza el óxido de cromo como catalizador, es decir como agente que impide unir nuevamente las moléculas del gas que ya se han separado a causa de la acción de la DBD; en este sentido el recubrir el electrodo interno con cromo hace más eficiente el proceso; en la figura 6 se aprecia el electrodo interno.

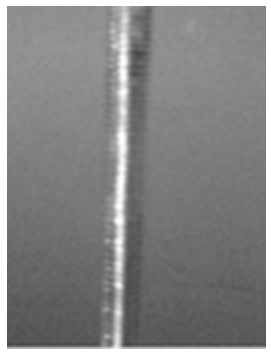

Figura 6. Varilla cromada que sirve como electrodo interior

\subsubsection{Dieléctrico}

Como elemento que actúa a manera de dieléctrico, se adaptó un tubo de alúmina $\left(\mathrm{Al}_{2} \mathrm{O}_{3}\right)$ del $99 \%$ de pureza, de $21,5 \mathrm{~cm}$ de largo $30 \mathrm{~mm}$ de diámetro exterior y 2,2 $\mathrm{mm}$ de espesor. Este material presenta buena estabilidad mecánica durante la generación de las descargas eléctricas, a diferencia de los tubos de vidrio pyrex o el quarzo, materiales también empleados en estas aplicaciones, los cuales tienden a fisurarse. En la figura 7 se muestra el tubo de alúmina utilizado como dieléctrico. Tubos de dimensiones similares han sido reportados en otros trabajos (Sarmiento et al., 2007; Sazal et al., 2011; Sentek et al., 2009).

\subsubsection{Tapas laterales}

Son dos elementos que se adaptan en los costados laterales del reactor cilíndrico, teniendo como 


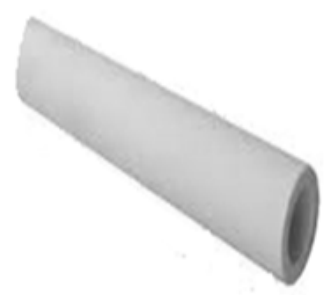

Figura 7. Tubo de Alúmina usado como barrera dieléctrica, sobre el cual se adapta el electrodo exterior

propósito el acople de la línea de gases con la cámara del reactor; lo anterior se lleva a cabo mediante acoples rápidos. Estas tapas se acoplan al tubo de cerámica mediante o-rings, para mantener herméticamente sellada la cámara de descarga, y además constituyen el soporte del electrodo interior de alta tensión, concéntrico al reactor. Las tapas se fabricaron en teflón, material con las propiedades adecuadas para soportar las temperaturas que se generan durante la descarga. En la figura 8 se muestran las tapas utilizadas.

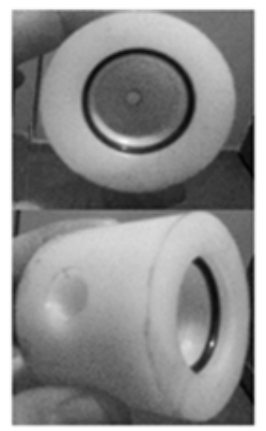

Figura 8. Tapas laterales fabricadas en teflón

En la figura 9 se muestra el reactor completamente ensamblado.

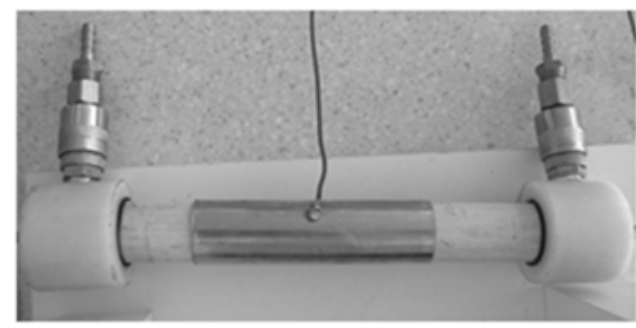

Figura 9. Reactor para experimentación con DBD

\subsection{Requerimientos y selección de la fuente de poder}

Para la obtención de plasma estable con las descargas de barrera de dieléctrico se necesita suministrar potencia con ondas de tensión elevada. Así es posible generar una descarga luminiscente y sostenerla a un nivel de tensión y corriente adecuado para evitar que se extinga el plasma (Figueroa, 2010 p. 37).

Algunas investigaciones relacionadas con la DBD, utilizaron fuentes de poder cuyas tensiones variaron entre 10 y $30 \mathrm{kv}$ y frecuencias de entre 6 y $30 \mathrm{kHz}$ (Kyung et al., 2012; Indarto, 2007; Sarmiento et al., 2007; Chong-Lin et al., 2008; Sazal et al., 2011; Sentek et al., 2009). La información reportada en esas investigaciones permitió establecer los criterios mínimos para selección de la fuente de poder, dadas sus similitudes con las características del presente trabajo.

La fuente seleccionada corresponde a la referencia PWM500/DRIVE10 del fabricante Information Unlimited, la cual está diseñada para alimentar sistemas de generación de plasma con tecnologías de descarga de barrera dieléctrica y de efecto corona. La fuente emplea un controlador de alta frecuencia, permitiendo al usuario adecuar cargas capacitivas en el rango de $5 \mathrm{pF}$ hasta $1 \mu \mathrm{F}$; además maneja tensiones que van desde 0 a 40 $\mathrm{kVp}$-p y frecuencias que van de 0 a $60 \mathrm{kHz}$.

\subsection{Arreglo final y experimentación preliminar}

\subsubsection{Ensamble del sistema}

El reactor se adaptó sobre una estructura en madera que lo soporta, la cual permite utilizar otros reactores de diferentes dimensiones a fin de realizar experimentaciones con otros gases y en otras condiciones. En la misma estructura se implementó un espacio para la toma de datos de las magnitudes físicas características de la descarga, a saber: tensión, potencia, frecuencia, y temperatura. En la figura 10 se ilustra el sistema base para la experimentación.

\subsubsection{Energización del sistema y análisis comparativo de funcionamiento}

Al tener el sistema ensamblado se procedió a establecer su funcionalidad. Se realizaron tres 


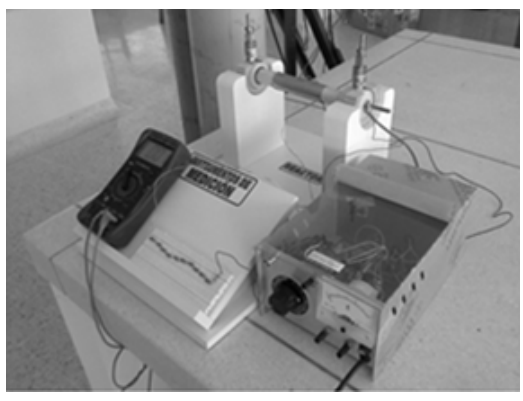

Figura 10. Sistema para experimentación con DBD

pruebas, las cuales buscan verificar que el sistema está en capacidad de ser usado para estudiar o transformar algunos gases entre los cuales están el metano $\mathrm{CH}_{4}$, el hidrógeno $\mathrm{H}_{2}$ y el dióxido de carbono $\mathrm{CO}_{2}$.

Se usó como gas de ensayo el aire, (78\% nitrógeno

\begin{tabular}{|c|c|c|c|}
\hline $\begin{array}{l}\text { PARÁMETROS DE } \\
\text { REFERENCIA }\end{array}$ & ENSAYO & $\begin{array}{c}\text { CONDICIONES EN EL } \\
\text { SISTEMA }\end{array}$ & $\begin{array}{c}\text { OBSERVACIONES Y/O } \\
\text { RESULTADOS }\end{array}$ \\
\hline $\begin{array}{l}\text { Tu y Whitehead }(2012) \text {, } \\
\text { trataron metano } \mathrm{CH}_{4} \text {, a } \\
\text { tensiones comprendidas } \\
\text { entre } 7.3 \text { y } 8.5 \mathrm{kV} \text { y } \\
\text { frecuencias de entre } 30 \text { y } \\
40 \mathrm{kHz} \text {. }\end{array}$ & 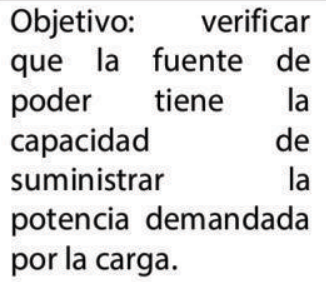 & $\begin{array}{l}\text { El reactor se mantuvo } \\
\text { energizado a través de } \\
\text { la fuente durante } 10 \\
\text { minutos manteniendo } \\
\text { un nivel de tensión de } \\
9.1 \mathrm{kV} \text { y una frecuencia } \\
\text { de } 25 \mathrm{kHz} \text {. }\end{array}$ & $\begin{array}{l}\text { La fuente de poder no } \\
\text { presentó calentamiento } \\
\text { durante la prueba. } \\
\text { No se generó plasma } \\
\text { visible. }\end{array}$ \\
\hline $\begin{array}{l}\text { Kyung y Gyu Lee, (2012), } \\
\text { transformaron } \mathrm{CH}_{4} \text { y } \mathrm{CO}_{2,} \\
\text { en } \mathrm{H} 2 \text { y } \mathrm{CO} \text { (syngas) a } \\
\text { tensión y frecuencia de } \\
6,6 \mathrm{kV} \text { y } 30 \mathrm{kHz} \text {. } \\
\text { Sarmiento et al., (2007) } \\
\text { generaron hidrógeno a } \\
\text { partir de hidrocarburos y } \\
\text { alcoholes a tensiones } \\
\text { comprendidas entre } 10 \text { y } \\
22 \text { kV y frecuencia de } \\
\text { entre } 1 \text { y } 6 \mathrm{kHz}\end{array}$ & $\begin{array}{l}\text { Objetivo: identificar } \\
\text { las condiciones en las } \\
\text { cuales se observa la } \\
\text { primera descarga } \\
\text { eléctrica en el gas. }\end{array}$ & $\begin{array}{l}\text { Se energizó el sistema } \\
\text { y se aumentó } \\
\text { gradualmente la } \\
\text { tensión y la frecuencia } \\
\text { encontrando una } \\
\text { primera descarga } \\
\text { eléctrica en el gas a } \\
\text { condiciones de } 12.31 \\
\text { kV y } 27.2 \mathrm{kHz} \text {. La } \\
\text { generación del plasma } \\
\text { se verificó a través de } \\
\text { un orificio realizado en } \\
\text { una de las tapas } \\
\text { laterales. }\end{array}$ & $\begin{array}{l}\text { Plasma visible color } \\
\text { violeta como descarga } \\
\text { filamentar. } \\
\text { No se presentó } \\
\text { calentamiento en la } \\
\text { fuente. } \\
\text { Distorsión de la señal de } \\
\text { tensión utilizando un } \\
\text { osciloscopio. }\end{array}$ \\
\hline $\begin{array}{l}\text { Lin Song et al., (2007) } \\
\text { trataron el dióxido de } \\
\text { nitrógeno a tensiones de } \\
\text { entre } 4 \text { a } 8.2 \mathrm{kV} \mathrm{y} \\
\text { frecuencias de entre } 13 \text { y } \\
17 \mathrm{kHz} \text {. }\end{array}$ & $\begin{array}{l}\text { Objetivo: identificar } \\
\text { las condiciones en las } \\
\text { cuales se genera } \\
\text { plasma estable }\end{array}$ & $\begin{array}{l}\text { Partiendo de las } \\
\text { condiciones del ensayo } \\
\text { anterior, se aumentó } \\
\text { gradualmente la } \\
\text { tensión encontrando } \\
\text { plasma estable en el } \\
\text { gas en una condición } \\
\text { de } 19.31 \text { kV v } 27.2 \text { kHz. }\end{array}$ & $\begin{array}{l}\text { Se genera plasma } \\
\text { estable de color violeta. } \\
\text { No se presentó } \\
\text { calentamiento en la } \\
\text { fuente. }\end{array}$ \\
\hline
\end{tabular}

Tabla1. Análisis comparativo de funcionamiento del sistema
$\mathrm{N}_{2}, 21 \%$ oxígeno $\mathrm{O}_{2}$ y $1 \%$ otros gases), dado que este gas tiene una tensión de ionización similar a la de los gases anteriormente mencionados. Los valores de tensión senoidal y de frecuencia tomados para la experimentación se definieron a partir de algunos trabajos de investigación reportados en la literatura, los cuales utilizan reactores con dimensiones similares al desarrollado en el presente trabajo.

En la tabla 1 se ilustra la experimentación realizada, mostrando en la primera columna los parámetros de referencia generales tomados del estado del arte; en las restantes columnas se aprecian los objetivos de cada ensayo, las condiciones tomadas para el sistema y los resultados obtenidos. 
En la figura 11 se muestra una imagen del plasma estable generado dentro del reactor, a condiciones de 19.31 kVy $27.2 \mathrm{kHz}$. En la imagen, el plasma se puede apreciar en la zona más clara que circunda el electrodo interno.

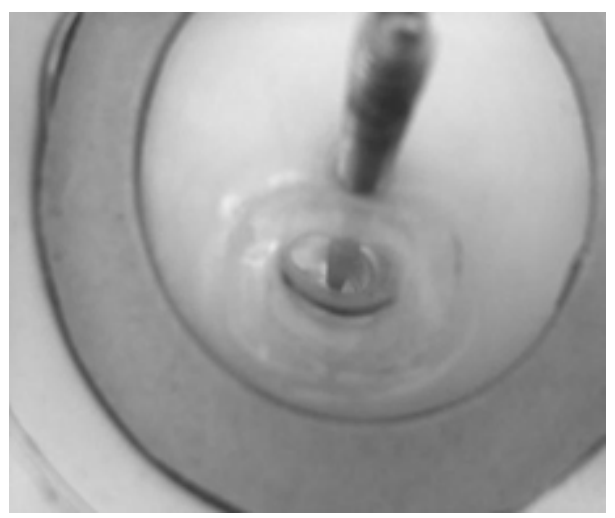

Figura 11. Imagen del plasma estable generado

\section{Conclusiones}

Se presentaron los fundamentos, requerimientos y algunas de las aplicaciones de la técnica de descarga de barrera de dieléctrico. Las bondades principales que este arreglo ofrece son por un lado las importantes aplicaciones en el manejo y tratamiento de gases, y por otro que la implementación de un sistema para experimentación no es complicada.

Se diseñó y construyó un sistema para experimentación con gases mediante la técnica de descarga de barrera de dieléctrico. Los materiales que se seleccionaron para la fabricación del reactor han tenido un buen desempeño en las condiciones de operación utilizadas.

Los valores de tensión y frecuencia, magnitudes características del plasma de la DBD, generados a través de aire, son similares a los reportados en trabajos de investigación que utilizan gases con tensiones de rompimiento o ionización similares a los del aire. Se demuestra con la experimentación que conseguir plasma estable en diferentes gases, con este sistema, es posible, pues se encontró plasma estable en aire a $19.31 \mathrm{kV}$ y frecuencia de $27.2 \mathrm{kHz}$; además, el sistema puede suministrar aún más energía aumentando la tensión y/o la frecuencia.

Los resultados de laboratorio presentados permitieron establecer la funcionalidad de la celda propuesta, de modo que a futuro podrán servir de base para el desarrollo de sistemas in situ en el tratamiento de gases como $\mathrm{CO}_{2}, \mathrm{SO}_{2} \mathrm{O}$ el $\mathrm{NO}_{2}$, entre otros.

\section{Referencias}

Chong-Lin S., Feng, B., Ze-Min, T., Fang Cheng, L., \& Qi-Fei H. (2008). Simultaneous removals of NOx, $\mathrm{HC}$ and PM from diesel exhaust emissions by dielectric barrier discharges. State Key Laboratory of Engines, Tianjin University, Tianjin. China.

Christensen, C. P. (1979). Pulsed transverse electrodeless discharge excitation of a $\mathrm{CO} 2$ laser. Applied Physics Letters, 34(3), 211-213.

Figueroa, A. (2010). Construcción y caracterización de un reactor de plasma de barrera dieléctrica para la producción de hidrógeno a partir de un gas metano (Tesis de maestría). Instituto Politécnico Nacional, Querétano México.

Fridman, A. (2008). Plasma Chemistry. 1 st ed. Cambridge University.

Givalov, J., \& Pietsch, V. G. (1992).Gas discharges and their applications. Great Britain. 552-555.

Hippler, R., Kersten. H., \& Scmidt, M. (2008). Low temperature plasmas. Fundamentals, technologies and techniques. 2 ed. Wiley-vch.

Indarto, A. (2008). Hydrogen production from methane in a dielectric barrier discharge using oxide zinc and chromium as catalyst. Journal of the Chinese Institute of Chemical Engineers, 39(1), 2328. 
Ishchenko, V. N., Lisitsyn, V. N., \& Sorokin, A. R. (1978). Excitation of high-pressure laser media by a discharge through an insulator. Quantum Electronics, 8(4), 453-457.

Kim, T. K., \& Lee, W. G. (2012). Reaction between methane and carbon dioxide to produce syngas in dielectric barrier discharge system. Journal of Industrial and Engineering Chemistry, 18(5), 17101714.

Kogelschatz, U., Eliasson, B., \& Hirth, M. (1988). Ozone generation from oxygen and air: discharge physics and reaction mechanisms.

Kogelschatz, U. (1988). Process Technologies for water treatment. S Stucki, plenum press. New York. 87-120.

Penetrante, B. M., Bardsley, J. N., \& Hsiao, M. C. (1997). Kinetic analysis of non-thermal plasmas used for pollution control. Japanese journal of applied physics, 36(7S), 5007.

Penetrante, B.M. Shultheis, S.E. (1993).Non thermal plasma techniques for pollution control. Series vol G34. Berlin.

Reitz, U., \& Fz Julich. (1992). Jul 2613. Concise synthesis of 1-deoxymannojirimycin. Bioorganic \& medicinal chemistry letters, 2(11), 1419-1422.

Rosocha, L. A. (1997). Plasma science and the environment. THStix editors. American Institute of of physics. New York. 261-298.

Ruan, R. R., Han, W., Ning, A., Chen, P. L., Goodrich, P. R., \& Zhang, R. (1999). Treatment of odorous and hazardous gases using non-thermal plasma. Journal of Advanced Oxidation Technologies, 4(3), 328-332.

Salge, J. (1996). Plasma-assisted deposition at atmospheric pressure. Surface and Coatings Technology, 80(1), 1-7.
Samojlovič, V. G., Gibalov, V. I., \& Kozlov, K. V. (1997). Physical Chemistry of the Barrier Discharge. DVSverlag GMBH. Düsserldorf.

Sarmiento, B., Brey, J. J., Viera, I. G., González-Elipe, A. R., Cotrino, J., \& Rico, V. J. (2007). Hydrogen production by reforming of hydrocarbons and alcohols in a dielectric barrier discharge. Journal of Power sources, 169(1), 140-143.

Sakal Kundu, K., Kennedy, E. M., Gaikwad, V. V., Molloy, T. S., \& Dlugogorski, B. Z. (2012). Experimental investigation of alumina and quartz as dielectrics for a cylindrical double dielectric barrier discharge reactor in argon diluted methane plasma. Chemical Engineering Journal, 180, 178-189.

Sentek, J., Krawczyk, K., Młotek, M., Kalczewska, M., Kroker, T., Kolb, T., ... \& Schmidt-Szałowski, K. (2010). Plasma-catalytic methane conversion with carbon dioxide in dielectric barrier discharges. Applied Catalysis B: Environmental, 94(1), 19-26.

Sillman, S., Logan, J. A., \& Wofsy, S. C. (1990). The sensitivity of ozone to nitrogen oxides and hydrocarbons in regional ozone episodes. Journal of Geophysical Research: Atmospheres (1984-2012), 95(D2), 1837-1851.

Song, C. L., Bin, F., Tao, Z. M., Li, F. C., \& Huang, Q. F. (2009). Simultaneous removals of $\mathrm{NO}<\mathrm{sub}>$ $\mathrm{x}</$ sub $>, \mathrm{HC}$ and $\mathrm{PM}$ from diesel exhaust emissions by dielectric barrier discharges. Journal of hazardous materials, 166(1), 523-530.

Sosa, R., Arnaud, E., Memin, E., \& Artana, G. (2009). Study of the flow induced by a sliding discharge. Dielectrics and Electrical Insulation, IEEE Transactions on, 16(2), 305-311. DOI :10.1109/TDEl.2009.4815157.

Tabata, N., Yagi, S., \& Hishii, M. (1996). Present and future of lasers for fine cutting of metal plate. Journal of materials processing technology, 62(4), 309-314. 
Tu, X., \& Whitehead, J. C. (2012). Plasma-catalytic dry reforming of methane in an atmospheric dielectric barrier discharge: Understanding the synergistic effect at low temperature. Applied Catalysis B: Environmental, 125, 439-448.
Weschler, C. J., Brauer, M., \& Koutrakis, P. (1992). Indoor ozone and nitrogen dioxide: A potential pathway to the generation of nitrate radicals, dinitrogen pentoxide, and nitric acid indoors. Environmental science \& technology, 26(1), 179184. 


\section{DISEÑO Y CONSTRUCCIÓN DE UNA BOBINA TESLA DE 1680 W, PARA LA ENSENAANZA DE CONCEPTOS BẤSICOS EN SISTEMAS ELÉCTRICOS DE POTENCIA}

\section{DESIGN AND CONSTRUCTION OF A TESLA COIL $1680 \mathrm{~W}$, AS A TOOL FOR TEACHING BASIC CONCEPTS IN ELECTRIC POWER SYSTEMS}

\section{Juan Carlos Castro Galeano ${ }^{1}$ María Luisa Pinto Salamanca² Maira Fernanda Amaya Quitián 3}

\section{Resumen}

La Bobina de Tesla es un tipo de transformador resonante, funciona a elevadas frecuencias y eleva la tensión, construida con dos bobinas acopladas eléctricamente en un núcleo de aire la cual produce efectos observables por el ojo humano como chispas y descargas eléctricas. Este artículo presenta el diseño y construcción de una Bobina de Tesla de $1680 \mathrm{~W}$ con una distancia de ruptura de $1 \mathrm{~m}$ de longitud, la cual puede ser empleada como herramienta didáctica y pedagógica para la enseñanza de los conceptos fundamentales de sistemas eléctricos de potencia, como: efecto corona, resonancia de circuitos RLC, campos eléctricos y magnéticos, transmisión de energía sin conductores y alta tensión.

Palabras clave: aislamiento eléctrico, efecto corona, descargas eléctricas, transformador deTesla.

\section{Abstract} Recibido: agosto 29 de 2014
Aceptado: octubre 24 de 2014

Tesla coil is a type of resonant transformer, which operates at high frequencies and raises the voltage; it's built with two coils electrically coupled into an air core which produces observable effects by the human eye as sparks and electrical discharges. This article presents the design and construction of a 1680 W Tesla coil with a rupture distance of one meter length. The coil could be used as a teaching learning tool in the teaching of the fundamental concepts of electrical power systems as: corona effect, resonance RLC circuits, electric and magnetic fields, energy transmission without conductors and high voltage.

Key words: electrical insulation, corona effect, electrical discharges, Tesla transformer.

\footnotetext{
1 Ingeniero Electricista, Magíster en Ingeniería. Docente Escuela de Ingeniería Electromecánica, Universidad Pedagógica y Tecnológica de Colombia, Facultad Seccional Duitama.

2 Ingeniera Electrónica, Magíster en Ingeniería. Docente Escuela de Ingeniería Electromecánica, Universidad Pedagógica y Tecnológica de Colombia, Facultad Seccional Duitama.

3 Ingeniera Electromecánica. Escuela de Ingeniería Electromecánica, Universidad Pedagógica y Tecnológica de Colombia, Facultad Seccional Duitama, E-mail:mafe78@gmail.com

1,2,3 Grupo de Investigación y Desarrollo en Sistemas Electromecánicos GridsE, E-mail:gridse.uptc@gmail.com
} 


\section{Introducción}

Nikola Tesla, un extraordinario ingeniero SerbioAmericano, descubrió el principio del campo magnético rotatorio en 1882 e inventó los motores de inducción y el sistema trifásico de generación y distribución de electricidad, fundamentando las bases de la energía eléctrica y los sistemas eléctricos de potencia. Gracias a esto, grandes cantidades de energía eléctrica pueden ser generadas y transmitidas a grandes distancias, desde las plantas generadoras hasta ciudades y centros de consumo.

Uno de sus inventos más memorables es la Bobina de Tesla, la cual es un transformador con núcleo de aire, formado por dos devanados, un primario y un secundario, los cuales son circuitos resonantes compuestos cada uno por una inductancia y una capacitancia que se encuentran a una resonancia específica (Rajvanshi, 2007, p.5). Con este dispositivo proyectaba transmitir la energía eléctrica sin necesidad de conductores, y aunque en esta época su invento no prosperó, los sistemas de comunicación inalámbrica y telefonía celular actuales se basan en este principio, actualmente el IEEE considera a Tesla uno de los doce apóstoles de la ciencia eléctrica y anualmente entrega un reconocimiento en su nombre en el campo de la potencia eléctrica (Vujic, 2001, p. 324).

Este dispositivo resulta ser muy útil a la hora de la enseñanza de diferentes conceptos del área eléctrica, como: efecto corona, frecuencia de resonancia en circuitos RLC, alta tensión, conducción e ionización del aire, transformadores elevadores, entre otros, y se convierte en una herramienta didáctica que a su vez es muy atractiva por sus impactantes destellos luminosos. Igualmente se puede emplear para realizar pruebas al aislamiento de equipos eléctricos (Phung, 1991; Pungsiri y Chotig, 2008; Sels et al., 2002).

Este artículo presenta el diseño, construcción y puesta a punto de una Bobina de Tesla de $1680 \mathrm{~W}$, con una distancia de ruptura de un metro de longitud; la bobina puede ser empleada como herramienta didáctica y pedagógica para la enseñanza de los conceptos fundamentales de sistemas eléctricos de potencia. A continuación se detallan el cálculo de los diversos componentes de la bobina de Tesla, junto con el proceso de fabricación y pruebas realizadas. Como resultado se obtuvo un arco de $40 \mathrm{~cm}$ y en la prueba con un tubo fluorescente, desde un metro de distancia, se iniciaron destellos luminosos en su interior.

\section{Materiales y métodos}

Para el diseño y construcción de los elementos de la bobina se empleó la metodología sugerida por, (Tilbury, 2008, p. 11-235), haciendo más comprensible su funcionamiento. A continuación se describen los diversos componentes de la bobina deTesla y su circuito equivalente.

\subsection{Circuito equivalente de la bobina de Tesla}

El circuito típico de la bobina de Tesla se presenta en la figura 1, en la cual:

T1: Transformador de alimentación elevador 120/15000V

R1: Resistencia limitadora

$\mathrm{Cp}$ :Condensador primario

Cp:Condensador secundario

Lp:Devanado primario

Ls: Devanado secundario

Spark Gap: Interruptor en aire

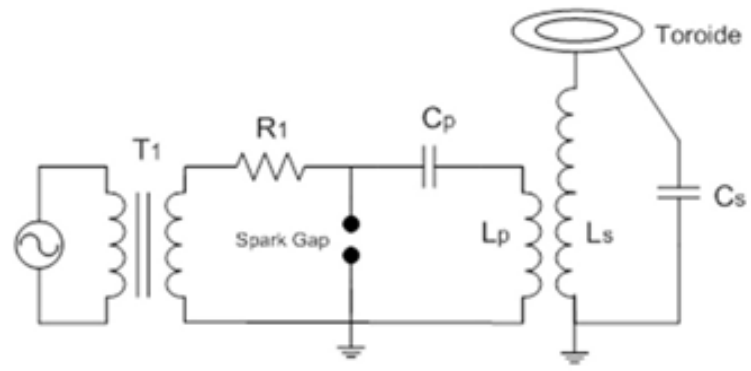

Figura 1. Circuito básico de la bobina de Tesla

El principio de funcionamiento de la bobina de Tesla se basa en un circuito resonante, donde la 
energía del transformador elevador T1 es empleada para cargar el condensador de alto voltaje $\mathrm{Cp}$. El alto voltaje al cual se ven sometidos los electrodos del spark gap, hace que se produzca en el aire a su alrededor una ionización generando un efecto de avalancha, que hace que el aire entre los gap's sea un conductor. Así, la energía almacenada en el condensador se descarga en el devanado primario Lp, en forma de oscilación de alta frecuencia, la cual es exactamente la frecuencia de resonancia entre los devanados primario y secundario de la bobina, con lo que se genera un alto voltaje en el terminal de descarga del devanado secundario, toroide, formando arcos de descarga hacia el electrodo de tierra. Este proceso de transferencia de energía de un circuito a otro continúa de forma cíclica, mientras se presenten rupturas en el sparkgap.

\subsection{Transformador de alimentación (T1)}

Se diseñó y construyó un transformador tipo seco con las siguientes características:

IAT: $130 \mathrm{~mA}$

VAT: $15000 \mathrm{~V}$

IBT: 16,67 A

VBT:120V

S: $2 \mathrm{kVA}$

P: $1950 \mathrm{~W}$

El devanado de alta tensión está formado por 26386 espiras, de alambre magneto calibre 31 AWG, distribuidas en ocho galletas y a su vez dividida en dos devanados para ser conectados en paralelo. El devanado de baja tensión posee 106 espiras en calibre 12 AWG en doble conductor, distribuidas en dos capas. El aislamiento del devanado de alta tensión se reforzó con resina Royal-POX y fibras Nómex de 0,25 mm entre galletas, ver figura 2 .

Considerando que el transformador va a estar sometido a esfuerzos eléctricos, éste se opera a una tensión de $12000 \mathrm{~V}$ para aumentar su vida útil. Para la selección del tipo de núcleo, construcción de los devanados y distancias de aislamiento y refrigeración se tomó la metodología del (MIT, E.E Staff, 2003, p. 151-285)

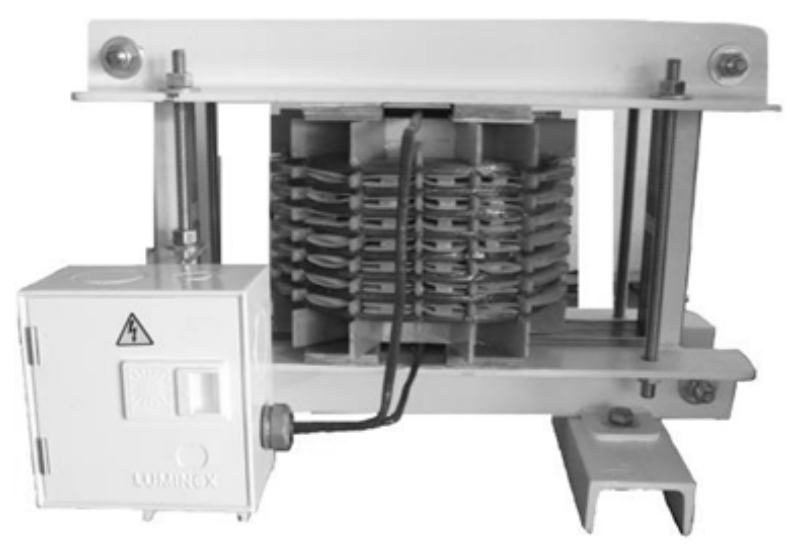

Figura 2. Transformador de alimentación T1

\subsection{Condensador Primario (Cp)}

Para la frecuencia de $60 \mathrm{~Hz}$, la tensión de $12000 \mathrm{Vy}$ la corriente de 0,13 A en el lado de AT del transformador $\mathrm{T} 1$, se calculó el valor de la capacitancia del condensador primario $\mathrm{Cp}$ empleando la ecuación (1)

$$
C p=\frac{I}{2 \pi \times f_{\text {linea }} \times V}
$$

Donde:

flinea: Frecuencia de línea, $\mathrm{Hz}$

V:Tensión, $\mathrm{V}$

I:Corriente, $\mathrm{A}$

$C p$ :Capacitancia primaria, $\mathrm{F}$

$$
C p=\frac{0,13}{2 \pi \times 60 \times 12000}=0,0287 \mu F
$$

El condensador se fabricó envolviendo dos flejes de aluminio de $0,2 \mathrm{~m} \times 2 \mathrm{~m}$ de longitud sobre un tubo de PVC, separados por un aislamiento compuesto por una fibra Tríplex en medio de dos fibras Nómex y dos fibras diamantadas, como se representa en la figura 3. 


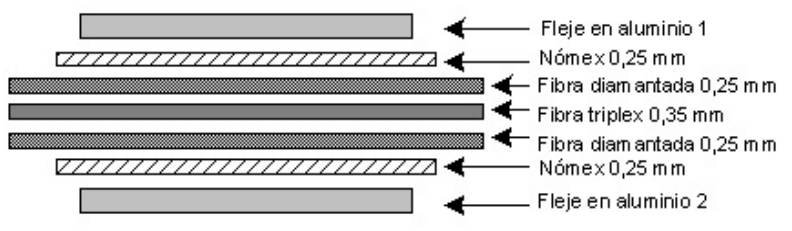

Figura 3. Esquema del aislamiento del condensador

El voltaje de ruptura estimado para el aislamiento empleado, se considera de $34 \mathrm{kV}$. Los voltajes de ruptura en $A C$ de los aislamientos empleados son: 2500V , 5000V y 19000 V para las fibras Nómex, Diamantada y Triplex respectivamente.

El proceso de fabricación del condensador se ilustra en la figura 4.
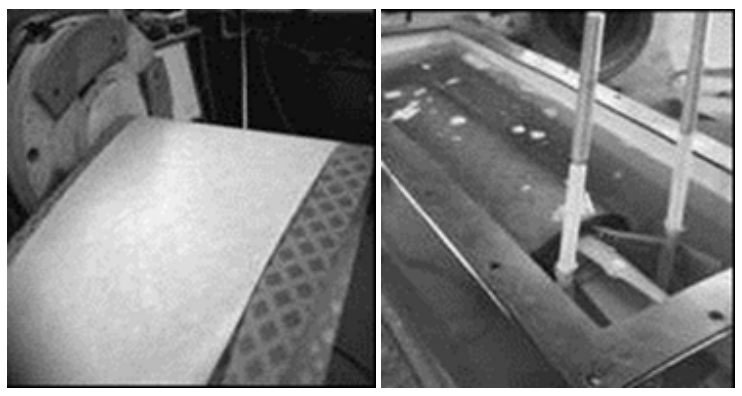

Figura 4. Fabricación del condensador $C p$

En la parte inicial de cada fleje se realizaron las salidas para ser conectadas a los bujes, realizados en teflón mediante maquinado en torno y en su interior con bulones de bronce. Una vez construido el condensador se sumergió en aceite dieléctrico para mejorar el aislamiento del mismo, verfigura 5 .

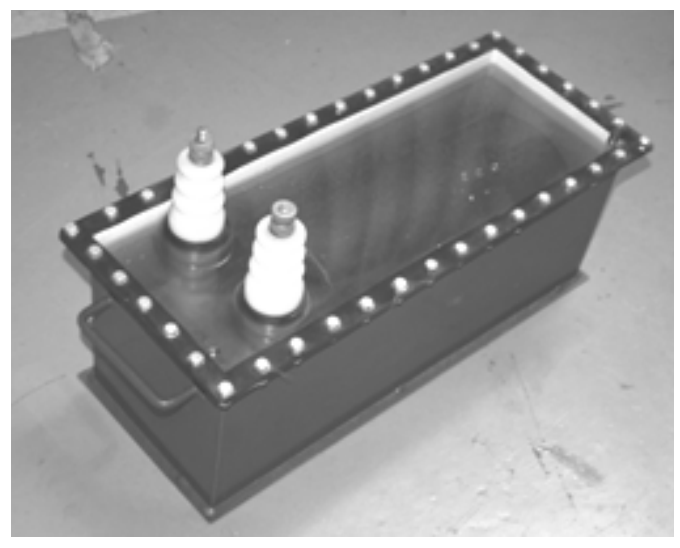

Figura 5. Condensador de alta tensión sumergido en aceite dieléctrico
Una vez construido $C P$, su valor medido empleando un multímetro FLUKE 187, fue de $0,017 \mu \mathrm{F}$, menor al valor calculado; por lo anterior fue necesario recalcular los demás componentes de la bobina.

\subsection{Bobina secundaria, Ls}

El secundario de la bobina de Tesla (alta tensión) se realizó en alambre magneto calibre 18 AWG con un total de 1100 espiras, enrolladas sobre un tubo de PVC de 16,81 cm de diámetro externo. Se posicionó de forma vertical y en la parte superior se ubicó el toroide en aluminio, para formar el terminal de alta tensión y a su vez distribuir uniformemente la capacitancia a tierra. El cálculo de la altura se realizó empleando la ecuación (2):

$$
H=N \times d
$$

Donde:

H: Altura de la bobina, $m$

$N$ : Número de espiras, 1100

$d$ : Diámetro del conductor de la bobina, $m$

El diámetro del alambre 18 AWG fue de 1,024 mm, portanto:

$$
H=1100 \times 0,001024=1,13 m
$$

Para el cálculo de la inductancia Ls se empleó la ecuación (3):

$$
L s=\frac{0,156 A^{2} N^{2}}{3,54 A+3,94 H} \times 0,001
$$

Donde:

A: Radio de la bobina, $\mathrm{cm}$

$N$ : Número de espiras de la bobina.

$\mathrm{H}$ : Altura de la bobina, $\mathrm{cm}$

$$
L s=\frac{0,16 \times 8,45^{2} \times 1100^{2}}{3,54 \times 8,45+3,94 \times 113} \times 0,001=29 \mathrm{mH}
$$


La capacitancia de la bobina en pF, se calculó empleando la ecuación (4), donde Hy A en metros

$$
\begin{aligned}
& C s=7,48 H+16,14 A+76,38 \sqrt{\frac{A^{3}}{H}} \quad(4) \\
& C s=7,48 \times 1,13+16,14 \times 0,0845+76,38 \sqrt{\frac{0,0845^{3}}{1,33}}=12 p F
\end{aligned}
$$

\subsection{Toroide}

Este terminal se ubica en la parte superior de la bobina secundaria de Tesla, comportándose como una capacitancia que completa el circuito de alta tensión, debido a que la autocapacitancia propia de la bobina no es suficiente y necesita ser reforzada. Cuando el circuito empieza a funcionar, la tensión es tan alta que llega un punto en que el terminal superior no puede contener mas carga y se produce la ruptura, la cual se manifiesta en un arco eléctrico.

En el diseño de esta bobina se seleccionó un toroide con diámetro exterior $\mathrm{D} 1=0,508 \mathrm{~m}$ y un diámetro del toroide $\mathrm{D} 2=0,1016 \mathrm{~m}$; para calcular su efecto hacia tierra, se empleó la ecuación (5).

$C_{t}=2,8 \times\left(1,2781-\frac{D_{1}}{D_{2}}\right) \times \sqrt{\frac{2 \pi^{2} \times\left(D_{1}-D_{2}\right) \times \frac{D_{2}}{2}}{4 \pi}}$

Para la elaboración del toroide se analizaron diferentes materiales, pero debido a que es recomendable emplear una superficie lisa, se optó por realizarlo en aluminio, para lo cual fue necesario construir un molde en madera para su fundición y su posterior mecanizado conservando sus dimensiones, figura 6. Una vez fabricado el toroide, éste fue ubicado sobre la bobina y se midió su capacitancia hacia tierra con un equipo DOBLE M4000, presentando un valor de 33,41 pF.

\subsection{Bobina primaria Lp}

Para el diseño de la bobina primaria, de baja tensión, se calculó la frecuencia de resonancia. Cuando los circuitos de alta y baja tensión

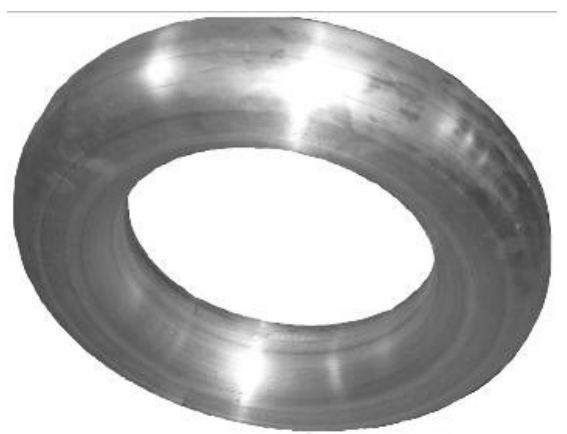

Figura 6. Toroide en aluminio fundido

alcanzan la misma frecuencia, se eleva la tensión en la bobina secundaria, produciendo la ionización del aire y los arcos eléctricos, desde el toroide a los elementos conectados a tierra, con una distancia menor a $1 \mathrm{~m}$ desde el mismo.

Empleando la ecuación (6) se calculó la frecuencia de la bobina secundaria fos, donde $L s$, en $[H]$ y Ctotalen [F].

$$
f_{o s}=\frac{1}{2 \pi \times \sqrt{L_{s} \times C_{\text {total }}}}
$$

Donde Ctotal considera la capacitancia del toroide de la bobina secundaria y el transformador T1, valor medido empleando equipo M4000:

$$
f_{\text {os }}=\frac{1}{2 \pi \times \sqrt{29 \times 10^{-3} \times 111,53 \times 10^{-12}}}=89,8 \mathrm{kHz}
$$

Para el cálculo de la resonancia de la bobina de Tesla, se igualaron la frecuencia de la bobina secundaria fos y primaria fop, y a partir de esta igualdad se calculó la inductancia de la bobina primaria Lp empleando la ecuación (7), con $f_{o p}=$ $f_{o s}$ :

$$
\begin{gathered}
\frac{1}{2 \pi \times \sqrt{L_{s} \times C_{\text {total }}}}=\frac{1}{2 \pi \times \sqrt{L_{p} \times C_{p}}} \\
L_{p}=\frac{L s \times C_{\text {total }}}{C_{p}}(7) \\
L_{p}=\frac{29 \times 10^{-3} \times 111,53 \times 10^{-12}}{17 \times 10^{-9}}=185 \mu \mathrm{H}
\end{gathered}
$$


Con este valor se calculó de forma iterativa el número de espiras de la bobina primaria empleando las ecuaciones (8) y (9):

$$
L p=\frac{N^{2} \times R}{30 R-11 D i},[\mu \mathrm{H}]
$$

Donde

N: Número de espiras del devanado primario

$R$ :Radio externo de la bobina primaria, $\mathrm{cm}$

Di:Diámetro interno de la bobina primaria, $\mathrm{cm}$

$$
R=\frac{D i+N(w+S)}{5,08},[\mathrm{~cm}]
$$

Donde:

w: Radio medio geométrico, $\mathrm{cm}$

\section{S:Espacio entre espiras}

Asumiendo un espaciado entre espiras de 1,52 cm, un conductor rectangular de radio medio geomérico de $1,18 \mathrm{~cm}$ y un diámetro interno de 23 $\mathrm{cm}$ se encontró la configuración para la bobina primaria.

Número de vueltas: 18

Diámetro interno: $22,86 \mathrm{~cm}$.

Diámetro externo: $105,3 \mathrm{~cm}$.

Espacio entre espiras: $1,5 \mathrm{~cm}$.

Para la elaboración de la bobina primaria, se fabricaron separadores en baquelita en forma de peinilla, ubicando en cada ranura una espira. Se empleó un conductor rectangular de 2,9 x 11,45 $\mathrm{mm}$, de cobre aislado con cinta Kapton. La bobina fabricada se presenta en la figura 7.

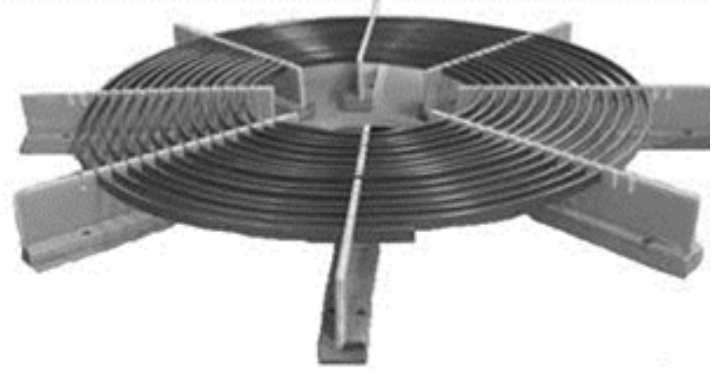

Figura 7. Bobina de baja tensión terminada

\subsection{Spark Gap}

El spark gap es el interruptor de alta potencia, encargado de iniciar la descarga en el circuito de alta tensión de T1 (primario de la bobina de Tesla). Está compuesto por unos electrodos separados una pequeña distancia en aire, el cual se ioniza cuando la tensión supera un umbral. Para su construcción se evaluaron varias alternativas, seleccionando uno del tipo dinámico y rotatorio, el cual depende de la velocidad de un motor para cambiar la frecuencia de resonancia de la bobina deTesla.

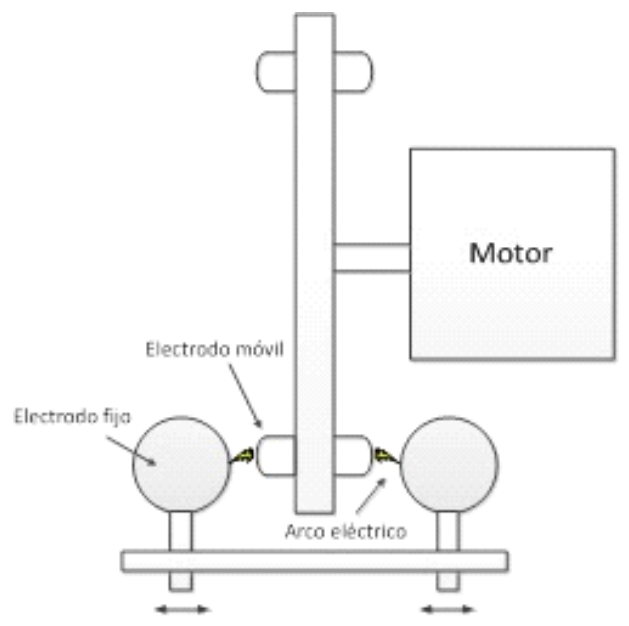

Figura 8. Interruptor en aire (spark gap)

En el eje del motor se acopló un disco aislante en baquelita sobre el cual se ubicaron cuatro electrodos iguales, y en la base se instalaron dos electrodos fijos a igual distancia de los electrodos móviles para que la ruptura fuera simultánea en ambos lados. Todos los electrodos se fabricaron en aluminio con terminación redondeada para controlar mejor la descarga simulando una ruptura entre electrodos planos. Los electrodos fijos se realizaron con un sistema de ajuste horizontal con el fin de variar la distancia y permitir el cambio de la tensión de ruptura, figura 8.

El motor fue soportado sobre una estructura metálica como presenta la figura 9, asegurando la 
altura adecuada para evitar saltos eléctricos desde los terminales energizados del spark gap hacia la estructura de la mesa.

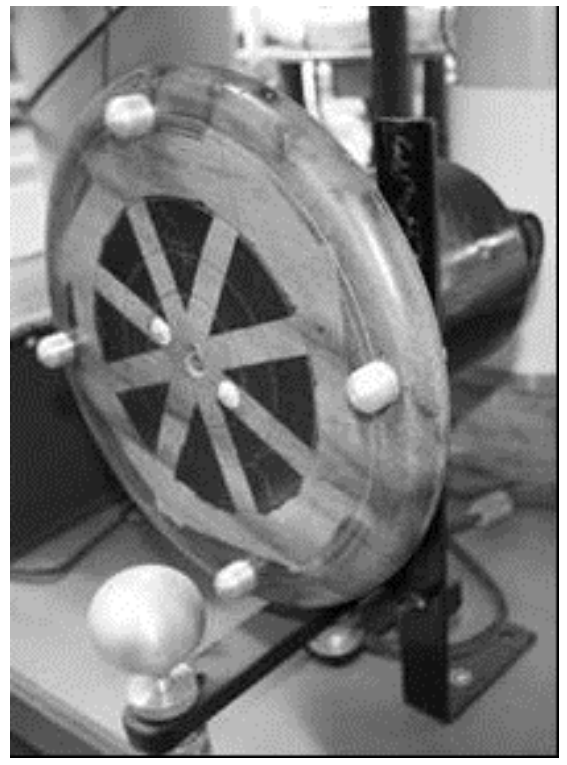

Figura 9. Spark gap construido

\subsection{Resistencia limitadora $\mathrm{R} 1$}

Para evitar corrientes elevadas en el transformador T1 en los instantes de operación del spark gap, se instaló una resistencia limitadora a la salida de su terminal de alta tensión. Esta resistencia se calculó simulando un corto en el lado de alta tensión de T1. Considerando que la corriente en este devanado es de $130 \mathrm{~mA}$, su impedancia de cortocircuito de $9,24 \%, 6,82 \mathrm{k} \Omega$ en las bases de $12 \mathrm{kV}$ y $2 \mathrm{kVA}$ y empleando el circuito de la figura 10 , se obtuvo una resistencia limitadora de 85,5 $\mathrm{k} \Omega$.

La resistencia limitadora se fabricó en agua empleando un tubo de PVC, como se muestra en la figura 11.

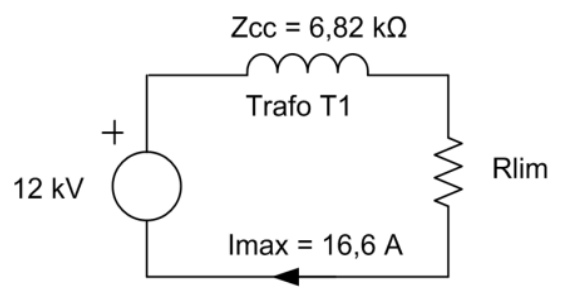

Figura 10. Cálculo de la resistencia limitadora R1

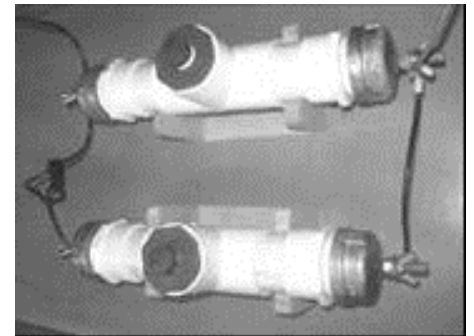

Figura 11. Resistencia limitadora

\section{Resultados y discusión}

Previo al ensamble final de la bobina, se verificó el correcto funcionamiento de sus componentes.

\subsection{Pruebas del transformadorT1}

Se realizó la prueba de resistencia óhmica de los devanados, resistencia de aislamiento y relación de transformación presentando resultados satisfactorios. Se midió la impedancia de corto circuito energizando el transformador por el lado de AT con en devanado de BT en corto, los valores medidos se presentan en la tabla 1. La impedancia de corto circuito se calculó empleando la ecuación (8):

$$
\begin{aligned}
& Z_{C C}=\frac{V_{\mathrm{Pr}}}{V_{n}} \times \frac{I_{n}}{I_{\mathrm{Pr}}} \times 100 \\
& Z_{C C}=\frac{435}{15000} \times \frac{130 \times 10^{-3}}{0,0408} \times 100=9,24 \%
\end{aligned}
$$

\begin{tabular}{cccc}
\hline \multicolumn{2}{c}{$\begin{array}{c}\text { Valores nominales lado } \\
\text { de AT }\end{array}$} & \multicolumn{2}{c}{$\begin{array}{c}\text { Valores medidos por } \\
\text { el lado de AT }\end{array}$} \\
\hline V, [V] & I, [mA] & V, [V] & I, [A] \\
15000 & 130 & 435 & 0,0408 \\
\hline
\end{tabular}

Tabla 1. Datos para el cálculo de la impedancia de corto circuito

Finalmente se realizó la prueba de factor de potencia y capacitancia del aislamiento como se sugiere en (Castro, 2009, p. 4), con el objetivo de medir las capacitancias del transformador T1 para realizar los ajustes en la frecuencia de resonancia en el circuito de la bobina de Tesla. La capacitancia AT contra BT y tierra, vista desde el circuito de la bobina deTesla, fue de 101,23 pF. 


\subsection{Ensamble final de la bobina de Tesla}

Se construyó una mesa de 1,2m x 1,2m, con los bordes metálicos y las estructuras horizontales en madera, para soportar todos los componentes de la bobina de Tesla. Las conexiones entre los elementos se realizaron lo más cortas posibles y se emplearon terminales para evitar desconexiones accidentales. El ensamble final se presenta en la figura 12.

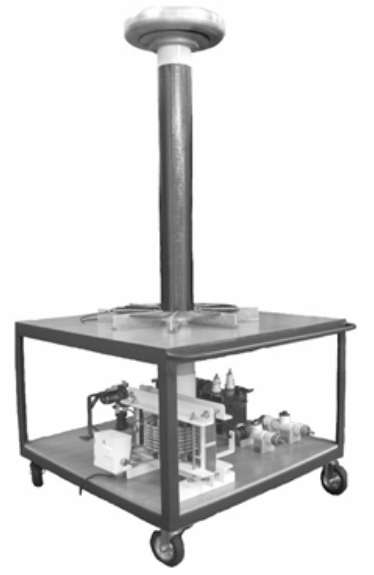

Figura 12. Bobina de Tesla ensamblada

\subsection{Pruebas finales}

Una vez ensamblada, se energizó la bobina y se obtuvo un arco de $40 \mathrm{~cm}$, como se muestra en la figura 13. La distancia de ruptura fue menor a la esperada, lo cual se debe en gran parte a las condiciones de temperatura, humedad y presión atmosférica, condiciones que disminuyen la capacidad de los equipos eléctricos de alta tensión.

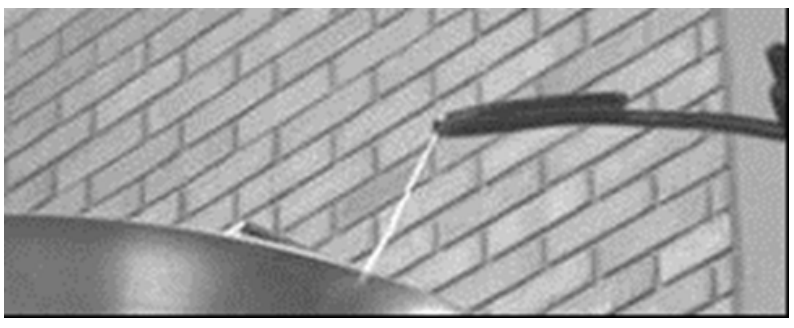

Figura 13. Arco generado

Se realizó la prueba con un tubo fluorescente y desde $1 \mathrm{~m}$ de distancia se iniciaron destellos de luz en su interior, los cuales aumentan en intensidad a medida que se acerca al toroide.

Dado el riesgo que existe al trabajar con sistemas de alta tensión, para realizar las conexiones del circuito de la bobina, es necesario que el equipo se encuentre desenergizado y el condensador sea descargado; la manipulación de alto voltaje y cargas eléctricas almacenadas pueden ser letales. Durante el funcionamiento de la bobina se producen campos eléctricos y magnéticos considerables, que pueden afectar el funcionamiento de equipos electrónicos sensibles; por ello se recomienda a las personas con marcapasos observar los destellos luminosos, producidos por la bobina, a una distancia considerable.

\section{Conclusiones}

Aunque el diseño de la Bobina de Tesla fue una actividad sencilla, su construcción fue compleja ya que debido a que sus componentes no se consiguen comercialmente, fue necesaria la construcción de cada uno de ellos. Una vez finalizada la construcción de todos sus elementos, se encontró que los valores medidos en algunos de ellos se alejaron de los esperados, desplazando de esta forma la frecuencia de resonancia de la bobina y disminuyendo los efectos luminosos, siendo necesario encontrar de forma experimental los ajustes y modificaciones para una nueva frecuencia realizando cambios en el número de espiras de la bobina de baja tensión, variando la velocidad del spark gap y cambiando los valores de la resistencia limitadora, la cual regula el tiempo de carga del condensador.

La bobina de Tesla aquí presentada permite un mejor análisis y comprensión de los conceptos fundamentales de los sistemas eléctricos de alta tensión, como: el efecto corona, resonancia en circuitos RLC, campos eléctricos y magnéticos y la transmisión de energía eléctrica sin conductores. Lo anterior debido a que estos conceptos se pueden explicar de forma práctica por medio de la 
Bobina de Tesla como herramienta didáctica de apoyo para la enseñanza de los fenómenos eléctricos: Además, podrá ser empleada para realizar pruebas en equipos aisladores y transformadores cuando son sometidos a sobretensiones transitorias.

Adicionalmente, el laboratorio de Electricidad de la Universidad Pedagógica y Tecnológica de Colombia, sede Duitama, cuenta con una bobina de Tesla para mejorar la enseñanza y la investigación del área eléctrica con la cual se beneficiarán todos los estudiantes de las áreas de electricidad de la Universidad ya que ninguna de las sedes cuenta con una bobina de este tipo.

\section{Agradecimientos}

Los autores agradecen a la Dirección de Investigaciones de la Universidad Pedagógica y Tecnológica de Colombia, por la financiación del proyecto: Diseño y construcción de una bobina tesla, para el laboratorio de electricidad de la UPTC sede Duitama; código SGI-873. Igualmente agradecen el apoyo técnico para la construcción de la bobina, a la empresa Industrias Explorer en Duitama, Boyacá.

\section{Referencias}

Castro, J. C. (2009). Pruebas de diagnóstico en transformadores. Congreso Internacional en Alta Tensión y aislamiento Eléctrico ALTAE 2009, Medellín noviembre de 2009.
M.I.T, E.E. STAFF (2003). Staff de Ingenieria Electrica (Electrical Engineering Staff) del Massachusetts Institute of Technology MIT. "Circuitos magnéticos y transformadores". España: Editorial Reverté.

Pungsiri, B., Chotig, S. (2008). The Compact Tesla Transformer for Testing Pin Insulator. International Conference on Condition Monitoring and Diagnosis, Beijing, China, April 2124.

Rajvanshi, A. K. (2007). Nikola Tesla - The creator of the Elecric Age. Resonance Journal of Science education, India: Bangalore.

Sels, T., Karas, J., Lopez-Roldan, J., Declercq, D., Dommelen, V., \& Belmans, R. (2002). Electrical Insulation Behaviour Subject to Fast Transients using a Tesla Transformer. Power and Energy Systems (PES 2002), Marina del Rey, USA, May 1315, 2002.

Tilbury, M. (2008). The Ultimate Tesla Coil Design and Construction Guide. United States: McGraw Hill.

Vujic, J. (2001). Nikola Tesla: 145 of Visionary Ideas. Congreso TELSLIKS 2001, Septiembre de 2001. (VUJIC, 2001, p. 324). 